(C) The Author(s), 2022. Published by Cambridge University Press on behalf of The Nutrition Society. This is an Open Access article, distributed under the terms of the Creative Commons Attribution-NonCommercial-NoDerivatives licence (http://creativecommons.org/licenses/by-nc-nd/ $4.0 /$, which permits non-commercial re-use, distribution, and reproduction in any medium, provided that no alterations are made and the original article is properly cited. The written permission of Cambridge University Press must be obtained prior to any commercial use and/ or adaptation of the article.

\title{
Hyperemesis gravidarum severity, enteral tube feeding and cardiometabolic markers in offspring cord blood
}

Kelly Nijsten ${ }^{1,2 *}$, Marjette H. Koot ${ }^{1}$, Joke M. J. Bais ${ }^{3}$, Carrie Ris-Stalpers ${ }^{4}$, Rik van Eekelen ${ }^{1}$, Henk A. Bremer ${ }^{5}$, David P. van der $\mathrm{Ham}^{6}$, Wieteke M. Heidema ${ }^{7}$, Anjoke Huisjes ${ }^{8}$, Gunilla Kleiverda ${ }^{9}$, Hinke Kruizenga ${ }^{10}$, Simone M. Kuppens ${ }^{11}$, Judith O. E. H. van Laar ${ }^{12}$, Josje Langenveld ${ }^{13}$, Flip van der Made ${ }^{14}$, Dimitri Papatsonis ${ }^{15}$, Marie-José Pelinck ${ }^{16}$, Paula J. Pernet ${ }^{17}$, Leonie van Rheenen-Flach ${ }^{18}$, Robbert J. Rijnders $^{19}$, Hubertina C. J. Scheepers ${ }^{20}$, Tatjana Vogelvang ${ }^{21}$, Ben W. Mol$^{22,23}$, Iris J. Grooten ${ }^{1}$, Tessa J. Roseboom ${ }^{1,2}$ and Rebecca C. Painter ${ }^{1}$

${ }^{1}$ Amsterdam UMC, University of Amsterdam, Department of Obstetrics and Gynaecology, Amsterdam Reproduction $\mathcal{E}$ Development Research Institute, Meibergdreef 9, Amsterdam, the Netherlands

${ }^{2}$ Department of Epidemiology and Data Science, Amsterdam UMC, University of Amsterdam, Amsterdam, the Netherlands

${ }^{3}$ Department of Obstetrics and Gynaecology, Noordwest Ziekenhuisgroep, Alkmaar, the Netherlands

${ }^{4}$ Laboratory of Reproductive Biology, Amsterdam University Medical Centers, University of Amsterdam, Amsterdam, the Netherlands

${ }^{5}$ Department of Obstetrics and Gynaecology, Reinier de Graaf Hospital, Delft, the Netherlands

${ }^{6}$ Department of Obstetrics and Gynaecology, Martini Hospital, Groningen, the Netherlands

${ }^{7}$ Department of Obstetrics and Gynaecology, Radboud University Medical Center, Nijmegen, the Netherlands

${ }^{8}$ Department of Obstetrics and Gynaecology, Gelre Hospital, Apeldoorn, the Netherlands

${ }^{9}$ Department of Obstetrics and Gynaecology, Flevo Hospital, Almere, the Netherlands

${ }^{10}$ Department of Nutrition and Dietetics, Internal Medicine, Amsterdam UMC, Vrije Universiteit Amsterdam, Amsterdam, the Netherlands

${ }^{11}$ Department of Obstetrics and Gynaecology, Catharina Hospital, Eindhoven, the Netherlands

${ }^{12}$ Department of Obstetrics and Gynaecology, Máxima Medical Center, Veldhoven, the Netherlands

${ }^{13}$ Department of Obstetrics and Gynaecology, Zuyderland Hospital, Heerlen, the Netherlands

${ }^{14}$ Department of Obstetrics and Gynaecology, Franciscus Gasthuis, Rotterdam, the Netherlands

${ }^{15}$ Department of Obstetrics and Gynaecology, Amphia Hospital, Breda, the Netherlands

${ }^{16}$ Department of Obstetrics and Gynaecology, Scheper Hospital, Emmen, the Netherlands

${ }^{17}$ Department of Obstetrics and Gynaecology, Spaarne Gasthuis, Haarlem, the Netherlands

${ }^{18}$ Department of Obstetrics and Gynaecology, OLVG, Amsterdam, the Netherlands

${ }^{19}$ Department of Obstetrics and Gynaecology, Jeroen Bosch Hospital, 's-Hertogenbosch, the Netherlands

${ }^{20}$ Department of Obstetrics and Gynaecology, Maastricht University Medical Center, Maastricht, the Netherlands

${ }^{21}$ Department of Obstetrics and Gynaecology, Diakonessenbuis, Utrecht, the Netherlands

${ }^{22}$ Department of Obstetrics and Gynaecology, Monash University, Clayton, VIC, Australia

${ }^{23}$ Aberdeen Centre for Women's Health Research, Institute of Applied Health Sciences, School of Medicine,

Medical Sciences and Nutrition, University of Aberdeen, Aberdeen, UK

(Submitted 6 July 2021 - Final revision received 18 January 2022 - Accepted 16 February 2022 - First published online 24 February 2022)

Abstract

This study aimed to investigate the association between hyperemesis gravidarum (HG) severity and early enteral tube feeding on cardiometabolic markers in offspring cord blood. We included women admitted for HG, who participated in the MOTHER randomised controlled trial

Abbreviations: FT4, free thyroxine; HG, hyperemesis gravidarum; IQR, interquartile range; RCT, randomised controlled trial; SGA, small for gestational age.

*Corresponding author: Kelly Nijsten, email k.nijsten@amsterdamumc.nl 
(RCT) and observational cohort. The MOTHER RCT showed that early enteral tube feeding in addition to standard care did not affect symptoms/ birth outcomes. Among RCT and cohort participants, we assessed how HG severity affected lipid, c-peptide, glucose and free thyroxine cord blood levels. HG severity measures were severity of vomiting at inclusion and 3 weeks after inclusion, pregnancy weight gain and 24-h energy intake at inclusion, readmissions and duration of hospital admissions. Cord blood measures were also compared between RCT participants allocated to enteral tube feeding and those receiving standard care. Between 2013-2016, 215 women were included: 115 RCT and 100 cohort participants. Eighty-one cord blood samples were available. Univariable not multivariable regression analysis showed that lower maternal weight gain was associated with higher cord blood glucose levels $(\beta:-0 \cdot 08,95 \% \mathrm{CI}-0 \cdot 16,-0 \cdot 00)$. Lower maternal weight gain was associated with higher Apo-B cord blood levels in multivariable regression analysis $(\beta:-0 \cdot 01,95 \% \mathrm{CI}-0 \cdot 02,-0 \cdot 01)$. No associations were found between other HG severity measures or allocation to enteral tube feeding and cord blood cardiometabolic markers. In conclusion, while lower maternal weight gain was associated with higher Apo-B cord blood levels, no other HG severity measures were linked with cord blood cardiometabolic markers, nor were these markers affected by enteral tube feeding.

\section{Key words: Cardiometabolic risk factors: CVD: Fetal nutrition disorders: Hyperemesis gravidarum: Fetal blood: Nutrition}

Hyperemesis gravidarum (HG) is a severe form of nausea and vomiting in pregnancy that affects up to $3.6 \%$ of pregnancies ${ }^{(1)}$. HG can lead to poor nutritional intake and significant weight loss ${ }^{(2,3)}$.

Maternal undernutrition during pregnancy has been demonstrated to lead to detrimental effects on offspring cardiometabolic disease risk in later life, for example, among adults exposed to maternal starvation while they were in utero during the Dutch Famine ${ }^{(4-6)}$. Early cardiometabolic disease markers are known to track through childhood into adulthood, enabling estimates of cardiometabolic diseases to be made in childhood, or even in infancy ${ }^{(7)}$. Cord blood lipid profile, glucose insulin metabolism and thyroid function have been demonstrated to provide such a window into the future ${ }^{(8-10)}$.

The nutritional intake of pregnant women with HG, which studies estimate to be as low as 450-1000 calories per day, falls drastically short of the recommendations ${ }^{(11)}$. Effects of HG on offspring's health in later life therefore seem likely but have sparsely been studied ${ }^{(12)}$. In the limited literature available on the topic of long-term effects of HG on offspring, a wide range of HG definitions and outcomes were employed, and little information was collected on the role of maternal nutrition ${ }^{(13-16)}$. This is of particular relevance as nutritional supplementation is not generally included in HG clinical management plans ${ }^{(17)}$. The role of maternal nutrition in HG or the role of HG disease severity on offspring health in later life has not been investigated, nor has the ability of nutritional supplementation to amend such effects.

Therefore, in this study, we aimed to investigate whether HG severity could affect cardiometabolic markers in offspring cord blood, as well as investigating the effect of early enteral tube feeding on these markers.

\section{Methods}

\section{Study design}

For this study, we used data from the Maternal and Offspring outcomes after Treatment of HyperEmesis by Refeeding (MOTHER) study ${ }^{(18)}$. The MOTHER trial, a multicentre open-label randomised controlled trial (RCT), aimed to evaluate the effect of early enteral tube feeding in addition to standard care for women hospitalised with HG. Beside this RCT, an observational cohort was assembled of eligible women who declined participation in the RCT but did consent to cohort participation. For more detailed information about the MOTHER study methodology, we refer to the corresponding study protocol and earlier published work ${ }^{(18,19)}$. Participants of both RCT and cohort were asked to provide informed consent for collection of maternal and umbilical cord blood for biobanking. The MOTHER study was registered at www.trialregister.nl (NTR4197) and approved by the research ethics committee of the Amsterdam UMC, location AMC (NL41011.018.12).

\section{Population}

Women admitted to hospital for HG between 5 and 20 weeks gestation were included in nineteen different hospitals in the Netherlands: three university medical centres and sixteen general hospitals that collaborated within the Dutch Consortium for studies in Obstetrics and Gynaecology. In our study, HG was defined as excessive vomiting necessitating hospital admission before 20 weeks gestation, and in the absence of any other causes of vomiting. Exclusion criteria were molar or non-vital pregnancies, maternal HIV, any contra-indication for enteral tube feeding and age $<18$ years. Eligible participants were identified by local staff of participating hospitals. Multiple pregnancies were excluded for the present cord blood study, because of the possibility that this could have independently affected offspring cord blood measures.

\section{Data collection}

Medical information was obtained from medical files by trained research staff and reported in a case report form. This case report form included detailed information about demographic characteristics as age, parity, gestational age and medical history as well as information about pregnancy and delivery. Preterm birth was defined as birth before the 37 th week of gestation. Neonates were considered small for gestational age (SGA) if their birth weight was below the 10th percentile according to the Dutch reference curves for birth weight by gestational age ${ }^{(20)}$. Pre-pregnancy weight, height, ethnicity and education level at baseline were self-reported. Pre-pregnancy BMI was calculated. Ethnicity was based on the country of birth of the participant's mother and for this study defined as Western (including participants from Organisation for Economic Co-operation and 
Development countries) or non-Western ${ }^{(21)}$. Education level was defined as the highest completed education: primary or secondary school $v$. higher education. Weight (in $\mathrm{kg}$ ) at inclusion was measured by hospital staff. Participants also recorded a weekly diary until 20 weeks gestation including symptom severity, maternal weight and a comprehensive dietary intake list. Symptom severity was measured by a validated questionnaire: the Pregnancy Unique Quantification of Emesis and nausea (PUQE-24) score; higher PUQE-24 scores indicate more severe symptoms and have previously been associated with a lower nutritional intake $\mathrm{e}^{(3,22)}$.

\section{Data collection on nutritional intake}

MOTHER participants kept a weekly self-reported, 24-h food diary, as shown in online Supplementary Figure A1. The food diary consisted of a list of pre-specified food products, with the ability to specify non-listed food products among 'others'. Intake of food products could be filled in millilitres as well as in pieces at six different moments. In collaboration with a dietician, databases of the Dutch National Institute for Public Health and the Environment (RIVM) were used to determine standard portion sizes and the corresponding energy content (kcal) of reported food products (online Supplementary Table A1) ${ }^{(23-25)}$. Eventually, a 24-h energy intake was calculated per women by adding up energetic quantities of the consumed food products. More detailed information about how dietary intake was collected and energy intake was calculated is shown in online Appendix A. According to the Dutch guidelines, a 24-h energy intake of at least the recommended $7824 \mathrm{~kJ}$ (1870 kcal) was considered normal for pregnant women ${ }^{(26)}$. This is based on $85 \%$ of the average daily energy intake in Dutch pregnant women, which is advised to be used as cut-off for the recommended daily intake by the Netherlands Nutrition Centre, since this has previously been shown to lead to a sufficient intake of essential recommended micronutrients, fibres and fatty acids ${ }^{(26,27)}$.

\section{Maternal and umbilical cord blood samples}

Random maternal blood samples were drawn during admission after study entry. Umbilical cord blood was taken after delivery, collected in a 20-ml syringe and then divided over five different blood aliquots of $4 \mathrm{ml}$ each: one EDTA tube, one heparin tube, one sodium fluoride tube and two serum tubes. Both maternal and cord blood samples were then transported and stored in one central laboratory at $-80^{\circ}$ until assayed. For this study, frozen stored samples were analysed in August 2019, 3-6 years after blood collection. We analysed Apo-A1, Apo-B, LDL, HDL, total cholesterol, TAG, c-peptide and free thyroxine (FT4) in serum cord blood samples. Cord blood glucose was analysed in sodium fluoride blood samples to prevent rapid glycolysis and therefore an underestimating of glucose levels. We also analysed maternal lipid profile and FT 4 at baseline in frozen stored maternal blood samples. Apo A1 and Apo B were measured on an Architect ci8200 with the use of immunoturbidimetric assay (Abbott Laboratories), with an intra-assay CV of respectively 0.8 and $1.4 \%$ and an inter-assay $\mathrm{CV}$ of 1.6 and $2.4 \%$. Total cholesterol was measured on a Roche Cobas 502 using photometric assay tests (Roche Diagnostics) with an intra-assay CV of $1.1 \%$ and an inter-assay CV of $1.6 \%$. HDL, TAG and glucose were measured on a Roche Cobas 702 using photometric assay (Roche Diagnostics) with intra-assay CV of $1 \cdot 0,0 \cdot 9$ and $0 \cdot 8 \%$ and inter-assay CV of $1 \cdot 3,2 \cdot 0$ and $1 \cdot 3 \%$, respectively. LDL was calculated by subtracting the HDL concentration and 0.45 times the TAG concentration of the total cholesterol concentration. C-peptide was measured with luminescence immunometric assay on an Advia Centaur analyser (Siemens Medical Solutions Diagnostics), with an intra-assay CV of $5 \%$ and an inter-assay of $7 \%$. FT4 was measured by a Roche Cobas e602 immuno-analyser (Roche Diagnostics) with an intra-assay $\mathrm{CV}$ of $2 \cdot 0 \%$ and an inter-assay of $2 \cdot 2 \%$.

\section{Determinants: measures of hyperemesis gravidarum severity}

We assessed HG severity as the severity of vomiting at inclusion (PUQE-24) and in the first 3 weeks after inclusion (average PUQE-24), the 24-h energy intake at inclusion, weight change at inclusion compared with pre-pregnancy weight, duration of hospital admissions, readmissions and readmission after the first trimester.

\section{Statistical analysis}

Due to the fact that early enteral tube feeding did not affect perinatal and maternal outcomes, we combined the RCT and cohort into one study population to assess associations between HG severity and cord blood outcomes ${ }^{(18)}$. SPSS Statistics 26.0 for Windows (IBM Corp.) was used for all analysis.

Descriptive statistics. Normally distributed continuous variables are presented as mean values and standard deviations, skewed distributions as medians with interquartile ranges (IQR) and categorical variables as frequencies with percentages. For FT4 levels, we were able to report how many neonates had an FT4 level below $(<12 \cdot 0 \mathrm{pmol} / \mathrm{l})$ or above $(>36 \cdot 0 \mathrm{pmol} / \mathrm{l})$ the national Dutch cord blood reference interval ${ }^{(28)}$. No corresponding reference intervals are available for any of the other endocrine or lipid measures in cord blood; therefore, we have reported these as continuous measures.

Associations between disease severity and cord blood outcomes. We performed univariable and multivariable linear regression analysis to assess associations between measures of HG severity and endocrine and lipid measures in cord blood. After assessing the effect of HG severity and other maternal and perinatal factors on endocrine and lipid measures in cord blood in univariate analyses, maternal and perinatal factors associated with endocrine and lipid measures in cord blood with a $P$-value below $0 \cdot 2$ on univariable analysis were considered for assessment in multivariable regression models. HG severity was kept as the primary determinant. In the multivariable model for the association of HG measures of severity on cord blood lipid measures, we adjusted for preterm birth, smoking, ethnicity, maternal diabetes (gestational, type I or II) and maternal lipid profile at study inclusion (i.e. maternal Apo-A1, Apo-B, HDL, LDL, total cholesterol or TAG, depending on which cord blood lipid profile measure was analysed). In the multivariable model 
for the association of HG measures of severity on cord blood glucose insulin metabolism (i.e. c-peptide and glucose), we adjusted for preterm birth, SGA, maternal age and pre-pregnancy BMI. In the multivariable model for the association of $\mathrm{HG}$ measures of severity on cord blood FT4, we adjusted for smoking, highest finished education and maternal FT4 levels at study inclusion. Associations between HG measures of severity and variables that were normally distributed were reported in differences $(\beta)$ and $95 \% 95 \%$ CI. Variables of which residuals were not normally distributed were logarithmically transformed to achieve normality before entering into the multivariable models. Subsequently, these variables were back-transformed and reported in proportionate differences $(\beta)$ and $95 \% \mathrm{CI}$ (in percentages).

Effect of nutritional supplementation. The effect of nutritional supplementation was evaluated in the RCT study population alone. We separately performed intention-to-treat, per protocol and 'as treated' analyses, in which the cord blood endocrine and lipid measures of patients in the RCT were reported according to allocation group to which they had been randomised (intentionto-treat), according to allocation and adherence to protocol (per protocol) or according to treatment with enteral tube feeding ('as treated'). For eligibility for the per protocol analysis, participants allocated to the intervention arm had to have received a nasogastric tube within $3 \mathrm{~d}$ after randomisation and continued for $7 \mathrm{~d}$ or longer, and participants allocated to standard care had to have received standard care alone in the first $3 \mathrm{~d}$ after randomisation. For the 'as treated' analysis, participants allocated to the intervention arm had to have received nasogastric tube within $7 \mathrm{~d}$ after randomisation and continued for $7 \mathrm{~d}$ or longer while participants allocated to standard care had to have received standard care alone in the first $7 \mathrm{~d}$ after randomisation. Chi-square test, Mann-Whitney U test and independent Student's $t$ test were used for statistical analyses.

Sensitivity analysis. A sensitivity analysis was performed to assess selective participation, by looking for differences in baseline characteristics and measures of HG severity between women with and without cord blood samples available.

Power calculation. A power calculation was performed for the original MOTHER study to determine the sample size of the RCT: 120 participants were randomised, based on finding a mean difference in birth weight of $200 \mathrm{~g}$ between the two intervention $\operatorname{arms}^{(18)}$. A post-hoc power calculation for this study was not performed, since there was already a set number of cord blood samples available together with the fact that post-hoc power calculations for multiple regression analysis are based on the coefficient of determinations $\left(R^{2}\right)$, which differ for each of the performed multivariable regression analyses.

\section{Results}

Between 2013 and 2016, 215 women participated in the MOTHER study: 115 participants in the RCT and 100 participants in the observational cohort. From the 210 singleton pregnancies, we had eighty-one cord blood samples available as shown in Fig. 1. Baseline characteristics and outcomes of women included in this study are shown in Table 1. The median 24-h energy intake at inclusion was $435 \mathrm{kcal}$ (IQR 152-1021). Sixty-two out of sixty-seven women (92.5\%) for whom 24-h energy intake was available had a 24-h energy intake that fell short of the recommended daily intake $(1870 \mathrm{kcal})^{(26)}$.

Sensitivity analysis showed that women with cord blood samples available had higher maternal TAG levels at inclusion (median 0.83; IQR 0.64-1.18 mmol/l) than women without cord blood samples available (median 0.75; IQR 0.61-1.02 mmol/l, $P$-value 0.045) (online Supplementary Table S1). Further baseline characteristics and outcome measures did not differ.

\section{Maternal and perinatal factors and their association with endocrine and lipid measures in cord blood}

Univariable regression analyses assessing associations between maternal and perinatal factors and each of the endocrine and lipid measures are presented in online Supplementary Table S2. Higher maternal pre-pregnancy BMI was associated with higher c-peptide levels in cord blood $(\beta 0.01,95 \% \mathrm{CI}$ $0 \cdot 00,0 \cdot 01)$. Smoking was associated with higher cord blood Apo-B ( $\beta 0.13,95 \%$ CI $0.01,0 \cdot 25)$ and higher LDL levels ( $\beta$ 0.46, 95\% CI 0.06, 0.87). Maternal gestational diabetes and diabetes type I or II during pregnancy were associated with higher Apo-B ( $\beta 0 \cdot 20,95 \%$ CI 0.06, 0.33), higher LDL ( $\beta 0 \cdot 77$, $95 \%$ CI $0.23,1.31)$ and higher total cholesterol levels in cord blood ( $\beta 0 \cdot 85,95 \%$ CI $0 \cdot 11,1 \cdot 60$ ). No associations were found between maternal age, ethnicity, highest completed educational level and maternal hypo- or hyperthyroidism and any of the endocrine and lipid measures in cord blood.

Regarding perinatal risk factors for endocrine and lipid measures in cord blood, we found that preterm birth was associated with higher Apo-B ( $\beta 0 \cdot 18,95 \%$ CI 0.05, 0.30) and higher total cholesterol levels in cord blood $(\beta 0 \cdot 75,95 \%$ CI $0 \cdot 17,1 \cdot 34)$. Preterm birth was also associated with lower cord blood LDL levels $(\beta-0 \cdot 66,95 \% \mathrm{CI}-1 \cdot 08,-0 \cdot 24)$. No associations were found between SGA or fetal sex and any of the endocrine and lipid measures in cord blood.

\section{Associations between measures of hyperemesis gravidarum severity and endocrine and lipid measures in cord blood}

Lipid profile. Lower maternal weight gain at study entry was associated with higher Apo-B levels $(\beta-0.01 \mathrm{~g} / \mathrm{l}$ per kg maternal weight gain, $95 \% \mathrm{CI}-0.02,-0.00, P=0.005)$ in multivariable linear regression analysis (Table 2). The 24-h energy intake at inclusion nor any of the other measures of HG severity was associated with lipid profile in cord blood.

C-peptide and glucose. Lower maternal weight gain at study entry was associated with higher glucose levels in cord blood in univariable linear regression analysis, $(\beta-0.08 \mathrm{mmol} / \mathrm{l}$ per kg maternal weight gain, $95 \% \mathrm{CI}-0 \cdot 16,-0 \cdot 00, P=0 \cdot 043)$, but the association was not sustained in multivariable models, as shown in Table 3. None of the measures of severity of HG was associated with c-peptide in cord blood. 


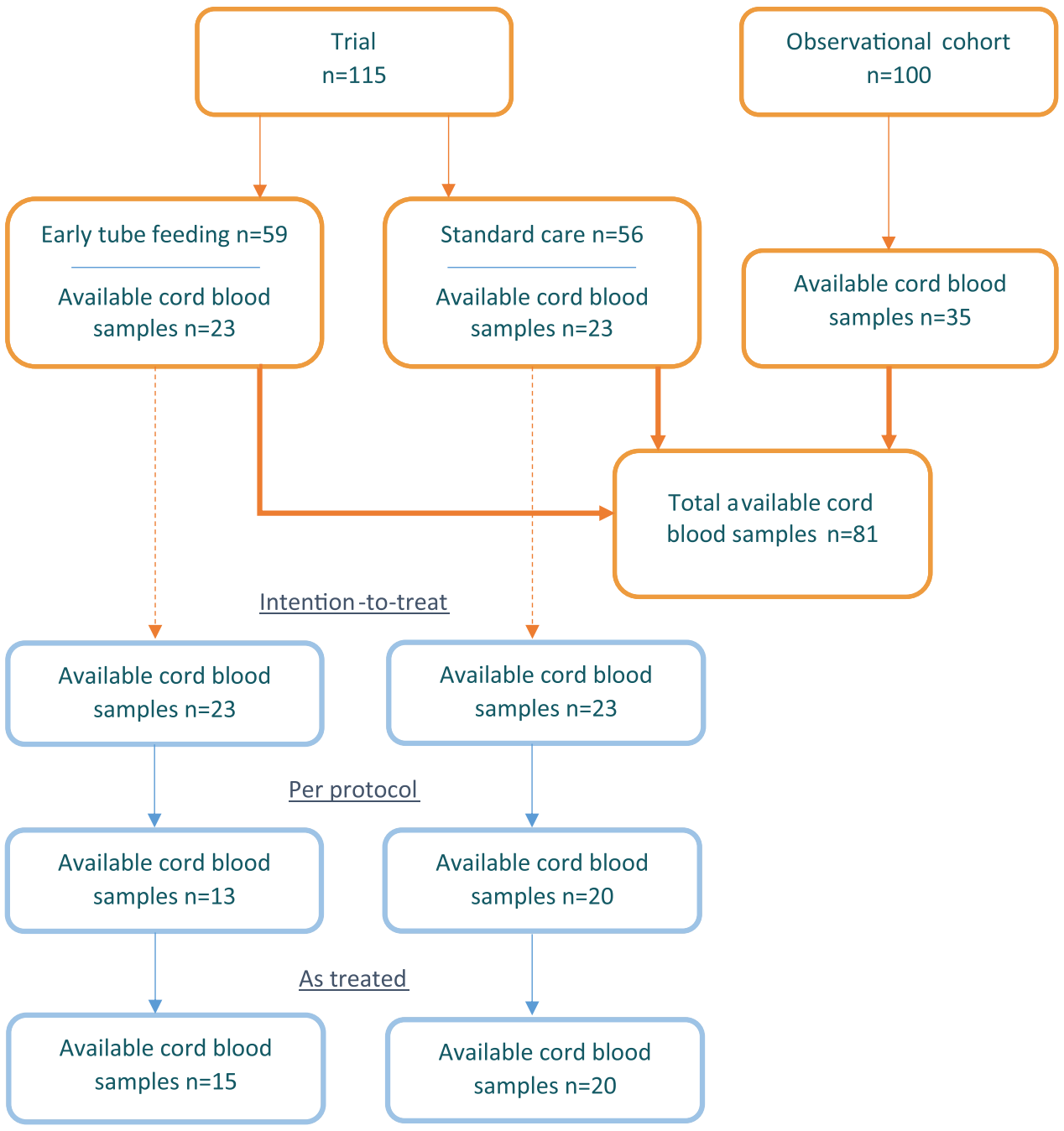

Fig. 1. Flowchart of available cord blood samples.

Free thyroxine. None of the measures of HG disease severity was associated with FT4 in cord blood (Table 3). One neonate had a FT4 cord blood level below the corresponding Dutch national reference interval $(<12.0 \mathrm{pmol} / \mathrm{l})$ of $10.4 \mathrm{pmol} / \mathrm{l}$. In this specific case, the mother had no medical history of hypo- or hyperthyroidism. She herself had normal thyroid stimulating hormone and FT4 concentrations at baseline ${ }^{(29)}$. Although birth weight was considered normal and the neonate was born at full term, the neonate was admitted to hospital postpartum due to suspicion of infection. No additional information on admission or further thyroid function measures was known. None of the neonates included in this study had a FT4 cord blood level above the corresponding Dutch national reference interval $(>36 \cdot 0 \mathrm{pmol} / \mathrm{l})$

The effect of early enteral tube feeding on endocrine and lipid measures in cord blood

In total, we had forty-six cord blood samples available for RCT participants. All included RCT participants with dietary intake at inclusion available (38/46) had a 24-h energy intake below the recommended daily intake of $1870 \mathrm{kcal}$. When comparing baseline characteristics, no differences were found in demographics, maternal blood measurements or perinatal outcomes, but few differences were found in measures of HG severity in intention-to-treat, per protocol and 'as treated' analysis. Participants who received early enteral tube feeding had a lower 24-h energy intake at inclusion than participants who received standard care, in both intention-to-treat (131 (IQR 43-417) v. 483 (IQR 283-1068) kcal) and per protocol analysis (82 (IQR 15-394) $v$. 605 (IQR 326-1129) kcal) (online Supplementary Table S3 and S4). In the as treated analysis, participants who received early enteral tube feeding had a lower 24-h energy intake at inclusion (76 (IQR 8-387) v. 605 (IQR 326-1129) kcal), higher vomiting scores at inclusion (PUQE-24:12.6 (sD 2.0) v. 10.3 (sD 3.3)), longer duration of admission to hospital (6.0 (IQR 3.0-10.0) v. 4.0 (IQR 2.3-5.0) days) and were readmitted more often $(53 \% v$ v $20 \%)$ than participants who received standard care (online Supplementary Table S5).

There were no differences in Apo-A1, Apo-B, HDL, LDL, total cholesterol, TAG, glucose, c-peptide and FT4 cord blood levels between offspring of participants who received early enteral 
Table 1. Baseline characteristics and outcome measures of women included in this study

(Number and percentages; median values and interquartile ranges)

$$
\frac{n 81}{n}
$$

\section{Demographics}

Age (years)

Mean

SD

Pre-pregnancy weight $(\mathrm{kg})$

Mean

SD

Pre-pregnancy BMI $\left(\mathrm{kg} / \mathrm{m}^{2}\right)$

SD

Non-Western ethnicity

Education level

Primary or secondary

Higher

Primigravida

Maternal diabetes (gestational, type I or II)

Maternal thyroid disease

Current smoker

Gestational age at onset of HG symptoms (weeks) Median

IQR

$n$

Gestational age at inclusion (weeks)

$$
\begin{aligned}
& \text { Median } \\
& \text { IQR }
\end{aligned}
$$

Measures of HG severity

Weight change $(\mathrm{kg})^{\star}$

$$
\text { Mean }
$$

SD

24-h energy intake at inclusion (kcal)

Median

IQR

Below recommended daily intake ( $<1870 \mathrm{kcal})$

PUQE-24 at inclusion

$$
\text { Mean }
$$

$$
\text { SD }
$$

Average PUQE-24 in the first 3 weeks after inclusion Mean

Total duration of hospital admissions (days) Median

\section{IQR}

Readmission

Readmission after the first trimester

Perinatal outcomes

Birth weight (grams)

Mean

SD

SGA (birth weight $<10$ th percentile)

Prematurity ( $<37$ weeks)

Apgar score $<7$ at $5 \mathrm{~min}$

Fetal sex (female)

\section{Maternal blood measurements}

Apo-A1 (g/l)

Apo-B (g/l)

$\mathrm{HDL}(\mathrm{mmol} / \mathrm{l})$

LDL (mmol/l)

Total cholesterol (mmol/l)

TAG (mmol/l)

Median

\begin{tabular}{|c|c|c|}
\hline $\begin{array}{r}-3.0 \\
3.8\end{array}$ & & $2.5 \%$ \\
\hline 435 & & $17 \cdot 3 \%$ \\
\hline $152-1021$ & & \\
\hline 62 & $76.5 \%$ & $17 \cdot 3 \%$ \\
\hline $10 \cdot 4$ & & $23.5 \%$ \\
\hline $3 \cdot 2$ & & \\
\hline $8 \cdot 7$ & & $27 \cdot 2 \%$ \\
\hline 2.9 & & \\
\hline $\begin{array}{l}5 \cdot 0 \\
3 \cdot 0-7 \cdot 0\end{array}$ & & $0.0 \%$ \\
\hline 27 & $33.3 \%$ & $0.0 \%$ \\
\hline 21 & $25.9 \%$ & $0.0 \%$ \\
\hline 3315 & & $0.0 \%$ \\
\hline 513 & & \\
\hline 6 & $7.5 \%$ & $1.2 \%$ \\
\hline 5 & $6.2 \%$ & $0.0 \%$ \\
\hline 2 & $2.5 \%$ & $1.2 \%$ \\
\hline 46 & $56.8 \%$ & $0.0 \%$ \\
\hline Mean & SD & \\
\hline 1.43 & 0.31 & $19.8 \%$ \\
\hline 0.80 & 0.25 & $19.8 \%$ \\
\hline 1.33 & 0.33 & $19.8 \%$ \\
\hline 2.43 & 0.83 & $19.8 \%$ \\
\hline $4 \cdot 21$ & 1.07 & $19.8 \%$ \\
\hline 0.83 & & $19.8 \%$ \\
\hline $0.64-1.18$ & & \\
\hline $19 \cdot 79$ & $5 \cdot 81$ & $19 \cdot 8 \%$ \\
\hline 0.90 & 0.18 & $7.4 \%$ \\
\hline 0.28 & 0.14 & $7.4 \%$ \\
\hline
\end{tabular}

IQR

FT4 (pmol/l)

Cord blood measurements

Apo-A1 (g/l)

Apo-B ( $g / l)$ 
Table 1. (Continued)

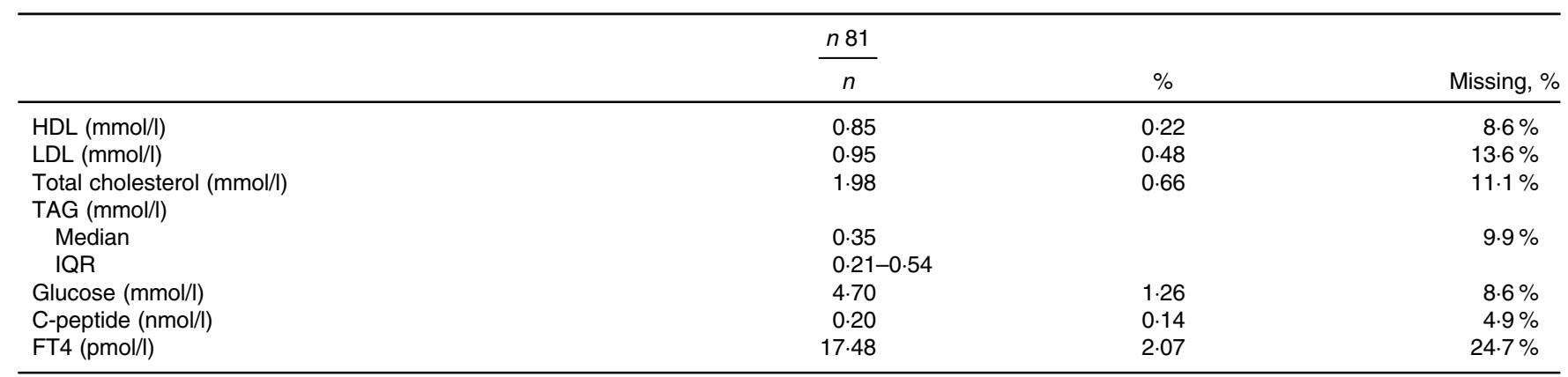

HG, hyperemesis gravidarum; FT4, free thyroxine; PUQE-24, 24-hour Pregnancy Unique Quantification of Emesis and nausea score: a higher PUQE-24 indicates more severe symptoms; SGA, small for gestational age.

*Weight change is weight at baseline minus pre-pregnancy weight and can be $<0$ if women lost weight and can be $>0$ if women gained weight.

tube feeding and offspring of participants who received standard care in intention-to-treat, per protocol and 'as treated' analysis (online Supplementary Tables S3-S5).

\section{Discussion}

\section{Main findings}

This small prospective cohort study was the first to find evidence that more severe HG could affect cardiometabolic disease markers in offspring at birth. We found that lower maternal weight gain at inclusion was associated with higher Apo-B and higher glucose levels in offspring cord blood. None of the other cord blood measures of possible cardiometabolic risk was affected by HG severity. We found no evidence that treatment by early enteral tube feeding improved offspring's cardiometabolic markers in cord blood.

\section{Strengths and limitations}

One of the strengths of our study is that detailed information about HG severity, including comprehensive nutritional information, was collected prospectively by trained research staff. With this study, we are addressing two of the top ten priority HG research questions that were recently identified by patients and clinicians, indicating the urgency of the need for guidance in the consequences of nutritional deficiency among women with $\mathrm{HG}^{(30)}$. This study can help meet patients' need for information on the long-term consequences of undernutrition associated with HG for their offspring - a source of significant maternal distress $^{(31)}$. Despite the small sample size, sensitivity analysis did not show any differences between MOTHER participants with and without cord blood samples available. We therefore think the likelihood of selection bias is small, which implies generalisability of our findings to all women with HG. The fact that we collected markers of possible future health by assessing cord blood, enabling estimates of increased cardiometabolic disease risk early in life, is another strength of this study ${ }^{(7)}$.

Cord blood samples were only available in eighty-one out of 215 participants (37\%). This small sample size limited our statistical power. Furthermore, there were missing data regarding weight change and dietary intake throughout pregnancy, after inclusion. For that reason, we were not able to assess gestational weight change and energy intake over time and include those measures as predictors in our regression analysis model. However, since PUQE-24 scores were more often reported in the first 3 weeks after inclusion, we were able to calculate average PUQE-24 scores and we also collected data of hospital admissions as measures of HG severity and did not find an association with endocrine and lipid measures in cord blood. While we used the validated PUQE-24 questionnaire ${ }^{(32,33)}$, information on nutritional intake was based on a non-validated 24-h food diary. In general however, food diaries have been considered as a reliable method to collect information on dietary intake, since they provide a detailed, prospective method with a low risk of recall bias ${ }^{(34,35)}$.

By design, our study did not include a control group of healthy pregnant women. Therefore, we are not able to comment on any possible changes in cardiometabolic markers in cord blood of HG cases compared with those in the offspring of healthy pregnant women. Finally, it is important to notice that, since we performed multiple statistical analyses, there is a considerable probability that some of our results were due to chance. When we applied the Bonferroni correction to our data, none of the associations we found remained significant (data not shown), which may support the notion that our findings were the result of chance ${ }^{(36)}$.

\section{Interpretation}

Our study showed that lower maternal weight gain at study inclusion was associated with higher Apo-B levels in cord blood. Apo-B is a protein that is attached to (very) low and intermediate density lipoproteins and which is used for transporting lipids around the body ${ }^{(37)}$. Apo-B has an important role in the pathogenesis of atherosclerosis and has previously been shown to be an independent and better predictor for CVD risk than $\mathrm{LDL}^{(37,38)}$. Birth weight can be a possible mediator for Apo-B levels in cord blood, although literature shows conflicting results ${ }^{(39-41)}$. We did not find an association between Apo-B cord blood levels and SGA neonates. Our sample size, however, prohibited any firm conclusions regarding infrequent outcomes including SGA (six out of eighty-one neonates) and preterm birth (five out of eighty-one neonates). Our study suggests that per extra kilogram maternal weight loss, Apo-B cord blood levels increase with $0.01 \mathrm{~g} / \mathrm{l}$. Considering the reference interval for Apo-B for infants younger than $14 \mathrm{~d}$ of $0 \cdot 10-0.67 \mathrm{~g} / \mathrm{l}$, the clinical relevance 
Table 2. Univariable and multivariable regression analysis assessing the association between measures of HG severity and lipid measures in cord blood (Coefficient values and $95 \%$ confidence intervals)

\begin{tabular}{|c|c|c|c|c|c|c|c|c|c|c|c|c|}
\hline & \multicolumn{6}{|c|}{ Apo-A1 (g/l) } & \multicolumn{6}{|c|}{ Apo-B (g/l) } \\
\hline & \multicolumn{3}{|c|}{ Model $1 \S$} & \multicolumn{3}{|c|}{ Model 2 § } & \multicolumn{3}{|c|}{ Model $1 \S$} & \multicolumn{3}{|c|}{ Model 2 § } \\
\hline & $\beta$ & $95 \% \mathrm{Cl}$ & $n$ & $\beta$ & $95 \% \mathrm{Cl}$ & $n$ & $\beta$ & $95 \% \mathrm{Cl}$ & $\boldsymbol{n}$ & $\beta$ & $95 \% \mathrm{Cl}$ & $n$ \\
\hline PUQE-24 at inclusion & -0.01 & $-0.02,0.01$ & 56 & -0.01 & $-0.02,0.01$ & 42 & -0.01 & $-0.01,0.00$ & 56 & -0.00 & $-0.01,0.01$ & 42 \\
\hline Average PUQE-24 first 3 weeks after inclusion & 0.00 & $-0.02,0.02$ & 54 & -0.01 & $-0.03,0.01$ & 38 & 0.01 & $-0.01,0.02$ & 54 & 0.00 & $-0.01,0.01$ & 38 \\
\hline Weight change $(\mathrm{kg}) \dagger$ & -0.00 & $-0.01,0.01$ & 72 & -0.01 & $-0.02,0.01$ & 50 & 0.00 & $-0.01,0.01$ & 72 & $-0.01^{*}$ & $-0.02,-0.00^{*}$ & 50 \\
\hline Energy intake at inclusion (kcal)‡ & -0.00 & $-0.01,0.01$ & 61 & 0.00 & $-0.01,0.01$ & 46 & -0.00 & $-0.01,0.00$ & 61 & 0.00 & $-0.00,0.00$ & 46 \\
\hline Duration of hospital admissions (days) & -0.00 & $-0.01,0.00$ & 74 & -0.00 & $-0.01,0.00$ & 50 & 0.00 & $-0.00,0.00$ & 74 & -0.00 & $-0.00,0.00$ & 50 \\
\hline Readmitted & 0.03 & $-0.06,0.12$ & 74 & 0.04 & $-0.05,0.13$ & 50 & 0.05 & $-0.02,0.11$ & 74 & 0.02 & $-0.04,0.08$ & 50 \\
\hline \multirow[t]{4}{*}{ Readmission after the first trimester } & -0.04 & $-0.14,0.05$ & 74 & -0.03 & $-0.14,0.08$ & 50 & -0.06 & $-0.13,0.02$ & 74 & -0.02 & $-0.09,0.05$ & 50 \\
\hline & \multicolumn{6}{|c|}{$\mathrm{HDL}(\mathrm{mmol} / \mathrm{l})$} & \multicolumn{6}{|c|}{ LDL (mmol/l) } \\
\hline & \multicolumn{3}{|c|}{ Model 1 § } & \multicolumn{3}{|c|}{ Model $2 \S$} & \multicolumn{3}{|c|}{ Model $1 \S$} & \multicolumn{3}{|c|}{ Model 2 § } \\
\hline & $\beta$ & $95 \% \mathrm{Cl}$ & $n$ & $\beta$ & $95 \% \mathrm{Cl}$ & $n$ & $\beta$ & $95 \% \mathrm{Cl}$ & $n$ & $\beta$ & $95 \% \mathrm{Cl}$ & $n$ \\
\hline PUQE-24 at inclusion & -0.01 & $-0.03,0.01$ & 55 & 0.00 & $-0.02,0.02$ & 41 & -0.02 & $-0.05,0.01$ & 52 & -0.02 & $-0.06,0.02$ & 38 \\
\hline Average PUQE-24 first 3 weeks after inclusion & -0.00 & $-0.03,0.02$ & 53 & -0.01 & $-0.03,0.02$ & 37 & 0.04 & $-0.02,0.09$ & 49 & 0.01 & $-0.04,0.06$ & 33 \\
\hline Weight change $(\mathrm{kg}) \dagger$ & 0.00 & $-0.01,0.02$ & 71 & -0.00 & $-0.02,0.01$ & 49 & 0.00 & $-0.03,0.04$ & 67 & -0.03 & $-0.05,-0.00$ & 45 \\
\hline Energy intake at inclusion (kcal) $\ddagger$ & 0.00 & $-0.01,0.01$ & 60 & 0.00 & $-0.01,0.01$ & 45 & -0.01 & $-0.03,0.01$ & 56 & -0.01 & $-0.02,0.01$ & 41 \\
\hline Duration of hospital admissions (days) & -0.00 & $-0.01,0.00$ & 73 & -0.00 & $-0.01,0.00$ & 49 & -0.00 & $-0.02,0.01$ & 69 & -0.00 & $-0.01,0.01$ & 45 \\
\hline Readmitted & 0.55 & $-0.14,0.08$ & 73 & 0.01 & $-0.11,0.14$ & 49 & 0.12 & $-0.13,0.36$ & 69 & 0.09 & $-0.12,0.29$ & 45 \\
\hline \multirow[t]{4}{*}{ Readmission after the first trimester } & -0.02 & $-0.13,0.10$ & 73 & -0.02 & $-0.17,0.12$ & 49 & -0.10 & $-0.36,0.16$ & 69 & -0.07 & $-0.17,0.32$ & 45 \\
\hline & \multicolumn{6}{|c|}{ Total cholesterol (mmol/l) } & \multicolumn{6}{|c|}{ TAG $\int(\mathrm{mmol} / \mathrm{l})$} \\
\hline & \multicolumn{3}{|c|}{ Model 1 § } & \multicolumn{3}{|c|}{ Model 2 § } & \multicolumn{3}{|c|}{ Model $1 \S$} & \multicolumn{3}{|c|}{ Model 2 § } \\
\hline & $\beta$ & $95 \% \mathrm{Cl}$ & $n$ & $\beta$ & $95 \% \mathrm{Cl}$ & $n$ & $\beta$ & $95 \% \mathrm{Cl}$ & $n$ & $\beta$ & $95 \% \mathrm{Cl}$ & $n$ \\
\hline PUQE-24 at inclusion & -0.03 & $-0.07,0.02$ & 54 & -0.03 & $-0.09,0.03$ & 40 & -2.57 & $-7 \cdot 13,2 \cdot 33$ & 54 & $-5 \cdot 16$ & $-11.31,1.41$ & 40 \\
\hline Average PUQE-24 first 3 weeks after inclusion & 0.04 & $-0.03,0.11$ & 51 & 0.01 & $-0.06,0.08$ & 35 & 2.53 & $-2 \cdot 86,8 \cdot 11$ & 52 & -1.29 & $-7.87,5.65$ & 36 \\
\hline Weight change $(\mathrm{kg}) \dagger$ & 0.01 & $-0.03,0.05$ & 69 & -0.03 & $-0.07,-0.01$ & 47 & 0.40 & $-3 \cdot 34,4 \cdot 19$ & 70 & -1.78 & $-6.29,3.05$ & 48 \\
\hline Energy intake at inclusion (kcal) $\ddagger$ & -0.01 & $-0.04,0.02$ & 58 & -0.00 & $-0.02,0.02$ & 43 & -0.70 & $-3.05,1.61$ & 59 & 0.60 & $-2 \cdot 27,3 \cdot 46$ & 44 \\
\hline Duration of hospital admissions (days) & -0.01 & $-0.03,0.01$ & 71 & -0.01 & $-0.02,0.01$ & 47 & 0.10 & $-1.49,1.82$ & 72 & -0.40 & $-2 \cdot 18,1 \cdot 31$ & 48 \\
\hline Readmitted & 0.13 & $-0.20,0.46$ & 71 & 0.14 & $-0.15,0.43$ & 47 & 30.60 & $-2 \cdot 26,75 \cdot 07$ & 72 & $29 \cdot 18$ & $-9.70,84.97$ & 48 \\
\hline Readmission after the first trimester & -0.16 & $-0.51,0.19$ & 71 & -0.02 & $-0.32,0.36$ & 47 & -14.62 & $-37.56,16.77$ & 72 & 2.43 & $-34 \cdot 88,61 \cdot 12$ & 48 \\
\hline
\end{tabular}

HG, hyperemesis gravidarum; PUQE-24, 24-hour Pregnancy Unique Quantification of Emesis and nausea score; the PUQE-24 score can range from 3 to 15 with a higher PUQE-24 indicating more severe symptoms.

* Significant associations with a $P$-value $<0.05$ are marked with an asterisk. † Weight change is weight at baseline minus pre-pregnancy weight and can be $<0$ if women lost weight and can be $>0$ if women gained weight. ₹ Energy intake at

inclusion is calculated over $24 \mathrm{~h}$, measured in kilocalories and divided by 100 for regression analysis. $\int=\log$ transformed, back transformed and expressed in \% of difference.
$\S$ Model 1: Univariable regression. Model 2: Multivariable regression, adjusted for preterm birth, smoking, ethnicity (Western or not), maternal diabetes (gestational, type I or II) and maternal lipid profile at inclusion. 
Table 3. Univariable and multivariable regression analysis assessing the association between measures of HG severity and endocrine measures in cord blood

(Coefficient values and $95 \%$ confidence intervals)

\begin{tabular}{|c|c|c|c|c|c|c|}
\hline & \multicolumn{6}{|c|}{ C-peptide (nmol/l) } \\
\hline & \multicolumn{3}{|c|}{ Model $1 \S$} & \multicolumn{3}{|c|}{ Model 2 § } \\
\hline & $\beta$ & $95 \% \mathrm{Cl}$ & $n$ & $\beta$ & $95 \% \mathrm{Cl}$ & $n$ \\
\hline PUQE-24 at inclusion & -0.00 & $-0.02,0.01$ & 58 & -0.01 & $-0.02,0.01$ & 56 \\
\hline Average PUQE-24 first 3 weeks after inclusion & 0.01 & $-0.01,0.02$ & 56 & 0.01 & $-0.01,0.02$ & 54 \\
\hline Weight change $(\mathrm{kg}) \dagger$ & -0.01 & $-0.01,0.00$ & 74 & -0.00 & $-0.01,0.01$ & 73 \\
\hline Energy intake at inclusion (kcal) $\ddagger$ & -0.00 & $-0.01,0.00$ & 63 & -0.00 & $-0.01,0.01$ & 61 \\
\hline Duration of hospital admissions (days) & -0.00 & $-0.01,0.00$ & 76 & 0.00 & $-0.00,0.00$ & 73 \\
\hline Readmitted & -0.01 & $-0.08,0.06$ & 76 & -0.00 & $-0.07,0.07$ & 73 \\
\hline \multirow[t]{4}{*}{ Readmission after the first trimester } & -0.02 & $-0.09,0.05$ & 76 & -0.02 & $-0.10,0.06$ & 73 \\
\hline & \multicolumn{6}{|c|}{ Glucose (mmol/l) } \\
\hline & \multicolumn{3}{|c|}{ Model $1 \S$} & \multicolumn{3}{|c|}{ Model 2 § } \\
\hline & $\beta$ & $95 \% \mathrm{Cl}$ & $n$ & $\beta$ & $95 \% \mathrm{Cl}$ & $n$ \\
\hline PUQE-24 at inclusion & -0.02 & $-0.12,0.10$ & 55 & -0.04 & $-0.17,0.08$ & 53 \\
\hline Average PUQE-24 first 3 weeks after inclusion & 0.06 & $-0.06,0.17$ & 54 & 0.06 & $-0.06,0.18$ & 52 \\
\hline Weight change $(\mathrm{kg}) \dagger$ & $-0.08^{\star}$ & $-0.16,-0.00^{*}$ & 71 & -0.08 & $-0 \cdot 16,0.01$ & 70 \\
\hline Energy intake at inclusion (kcal)‡ & 0.01 & $-0.04,0.06$ & 60 & 0.01 & $-0.04,0.07$ & 58 \\
\hline Duration of hospital admissions (days) & 0.01 & $-0.02,0.04$ & 73 & 0.01 & $-0.03,0.04$ & 70 \\
\hline Readmitted & 0.22 & $-0.39,0.83$ & 73 & 0.10 & $-0.54,0.74$ & 70 \\
\hline \multirow[t]{4}{*}{ Readmission after the first trimester } & 0.56 & $-0.09,1.21$ & 73 & 0.48 & $-0 \cdot 22,1 \cdot 19$ & 70 \\
\hline & \multicolumn{6}{|c|}{ FT4 (pmol/l) } \\
\hline & \multicolumn{3}{|c|}{ Model $1 \S$} & \multicolumn{3}{|c|}{ Model 2 § } \\
\hline & $\beta$ & $95 \% \mathrm{Cl}$ & $n$ & $\beta$ & $95 \% \mathrm{Cl}$ & $n$ \\
\hline PUQE-24 at inclusion & -0.05 & $-0.24,0.15$ & 45 & -0.19 & $-0.43,0.06$ & 31 \\
\hline Average PUQE-24 first 3 weeks after inclusion & 0.17 & $-0.07,0.41$ & 43 & 0.20 & $-0.09,0.50$ & 29 \\
\hline Weight change $(\mathrm{kg}) \dagger$ & 0.00 & $-0.14,0.14$ & 58 & 0.11 & $-0.08,0.29$ & 37 \\
\hline Energy intake at inclusion (kcal)‡ & 0.08 & $-0.01,0.17$ & 49 & 0.06 & $-0.04,0.17$ & 34 \\
\hline Duration of hospital admissions (days) & -0.01 & $-0.07,0.05$ & 60 & -0.01 & $-0.07,0.06$ & 37 \\
\hline Readmitted & -0.42 & $-1.55,0.71$ & 60 & 0.25 & $-1 \cdot 16,1 \cdot 65$ & 37 \\
\hline Readmission after the first trimester & 0.45 & $-0.76,1.66$ & 60 & -0.14 & $-1 \cdot 71,1.43$ & 37 \\
\hline
\end{tabular}

HG, hyperemesis gravidarum; FT4, free thyroxine; PUQE-24, 24-hour Pregnancy Unique Quantification of Emesis and nausea score; the PUQE-24 score can range from 3 to 15 with a higher PUQE-24 indicating more severe symptoms.

* Significant associations with a $P$-value $<0.05$ are marked with an asterisk. $†$ Weight change is weight at baseline minus pre-pregnancy weight and can be $<0$ if women lost weight and can be $>0$ if women gained weight. $\ddagger$ Energy intake at inclusion is calculated over $24 \mathrm{~h}$, measured in kilocalories and divided by 100 for regression analysis.

$\S$ Model 1: Univariable regression analysis. Model 2: Multivariable regression analysis for c-peptide and glucose was adjusted for preterm birth, small for gestational age, maternal age and pre-pregnancy BMI. Multivariable regression analysis for FT4 was adjusted for smoking, highest finished education and maternal FT4 levels at study inclusion.

of this difference, even in cases with severe weight loss, might be low ${ }^{(42)}$.

Currently, there is only a small body of evidence available describing the effects of HG on offspring's CVD risk in later life in comparison with that among offspring of uncomplicated pregnancies $^{(13-16)}$. None of these studies found differences in offspring lipid concentrations in childhood ${ }^{(13-16)}$. Two studies, however, did find that offspring born to women with HG were heavier and more adipose in childhood than offspring born to women without $\mathrm{HG}^{(14,16)}$, which can in line lead to increased Apo-B levels in later life and therefore affect offspring CVD risk $^{(43)}$. Taking our findings into account, differences in these studies' findings might be due to differences in HG severity with studies including women with a more severe HG being more likely to find associations than studies including women with a relatively mild HG. Another explanation for differences in results could be the sample size or, more important, the number of HG-exposed offspring included. Although Koot et al. ${ }^{(13)}$ included 6462 adolescents in their study, only forty-two had mothers with HG. Their study did not find any differences in cardiometabolic risk factors due to HG. Poeran-Bahadoer et $a l^{(16)}$ and Grooten et $a l^{(14)}$ included a far larger group of women with daily vomiting and severe weight loss (respectively 463 and 533 women) and found significant differences in anthropometric measures and blood pressure. However, neither study has prospectively collected information on HG diagnosis and treatment.

Our results show that a more severe HG, in terms of lower maternal weight gain at inclusion, was associated with higher glucose levels, in the absence of increased c-peptide levels in cord blood. These findings are in line with earlier studies: research from the Dutch famine showed an increased risk of developing diabetes in later life in offspring conceived during the famine and Ayyavoo et al. ${ }^{(15)}$ found a lower insulin sensitivity in offspring of women with HG in childhood ${ }^{(44,45)}$. Importantly, glucose levels in cord blood are likely highly reflective of maternal blood glucose concentrations ${ }^{(46)}$. This may point to an increase in maternal hyperglycaemia in more severe $\mathrm{HG}$ 
cases, which corresponds to literature linking maternal starvation to insulin resistance, which in turn could have independent effects on the chance of offspring adiposity and insulin resistance in later life ${ }^{(47)}$

While our study suggests that lower maternal weight gain in HG is associated with some increased markers of cardiometabolic disease risk for offspring, treatment by early enteral tube feeding, a treatment which could potentially restore nutritional intake, did not affect offspring's cardiometabolic markers in cord blood. Given the small sample size, with just forty-six women in RCT analyses, our statistical power was limited. Our study would have been able to detect only large mean differences based on treatment (for instance a mean difference of $0 \cdot 11 \mathrm{~g} / \mathrm{l}$ for Apo-B cord blood levels, based on using a two group $t$ test with $80 \%$ power and $5 \%$ two-sided significance level, assuming that the common standard deviation is $\left.0 \cdot 13 \mathrm{~g} / \mathrm{l}^{(39,41)}\right)$. Alternatively, it is also possible that women received tube feeding too late or for too short a period to have a beneficial effect on offspring cardiometabolic markers in cord blood. Other treatment aspects of interest, but out of scope for this study, include the impact of adequate antiemetic treatment on symptoms and maternal weight gain and are of interest for future studies on the efficacy of medications in the treatment of HG. For example, there is some evidence showing that starting antiemetic treatments early in pregnancy can reduce the overall severity of HG, which might have a beneficial effect on neonatal outcomes as well ${ }^{(48,49)}$.

\section{Conclusion}

Our study showed that lower maternal weight gain was associated with higher Apo-B levels in offspring cord blood, possibly indicating an increased cardiometabolic risk in later life, although this effect is modest and the clinical relevance remains unclear. None of the other measures of HG severity was associated with any of the other endocrine and lipid measures in cord blood, which could also mean that our results are based on coincidence. Additionally, our study provided insufficient evidence to show that early enteral tube feeding amended offspring outcomes. Larger and longer follow-up studies are necessary to further evaluate the possible negative impact of HG on offspring's long-term health and to assess the role of HG's disease spectrum or nutritional and other management options.

\section{Acknowledgements}

We thank all participating women of the MOTHER study and all staff who made this study possible, including staff from the Amsterdam UMC laboratory who helped us analysing frozen stored blood samples (Resources contributor role).

The MOTHER study was conducted with support of a research grant from North West Hospital Group, Alkmaar, the Netherlands (grant number 2013T085). The follow-up study analysing maternal and cord blood samples was conducted with support of a research grant from the Amsterdam Reproduction and Development (AR\&D) research institute, Amsterdam UMC, the Netherlands (project number 23346).

I. J. G., K. N., M. H. K., R. C. P. and T. J. R.: formulated research questions and designed the study; K. N. and R. C. P. carried out the study; J. M. J. B., C. R-S., H. A. B., D. Pvd. H., W. M. H., A. H., G. K., S. M. K., J. O. E.Hv. L., J. L., Fvd. M., D.P., M-J. P., P. J. P., LvR. F., R. J. R., H. C. J. S., T. V., B. W. M., M. H. K., I. J. G. and R. C. P.: performed recruitment and data collection of the original MOTHER study; H. K.: gave perspective on the interpretation of dietary results; K. N., supervised by R. C. P. and Rv. E.: analysed data; K. N. drafted the manuscript. All authors critically reviewed the manuscript and approved the final draft.

Dr. B.W. Mol was supported by a NHMRC investigator grant. Dr. B.W. Mol also reports consultancy for Guerbet and has received research funding from Guerbet, Ferring and Merck. Drs. K. Nijsten and all other authors report no conflict of interests.

\section{Supplementary material}

For supplementary materials referred to in this article, please visit https://doi.org/10.1017/S0007114522000587

\section{References}

1. Einarson TR, Piwko C \& Koren G (2013) Quantifying the global rates of nausea and vomiting of pregnancy: a meta analysis. J Popul Therapeut Clin Pharmacol 20, e171-83.

2. van Stuijvenberg ME, Schabort I, Labadarios D, et al. (1995) The nutritional status and treatment of patients with hyperemesis gravidarum. Am J Obstet Gynecol 172, 1585-1591.

3. Birkeland E, Stokke G, Tangvik RJ, et al. (2015) Norwegian PUQE (Pregnancy-Unique Quantification of Emesis and nausea) identifies patients with hyperemesis gravidarum and poor nutritional intake: a prospective cohort validation study. PLOS ONE 10, e0119962.

4. Roseboom TJ, van der Meulen JH, Osmond C, et al. (2000) Plasma lipid profiles in adults after prenatal exposure to the Dutch famine. Am J Clin Nutr 72, 1101-1106.

5. Roseboom TJ, van der Meulen JH, Osmond C, et al. (2000) Coronary heart disease after prenatal exposure to the Dutch famine, 1944-1945. Heart 84, 595-598.

6. Roseboom TJ, van der Meulen JH, Ravelli AC, et al. (2000) Plasma fibrinogen and factor VII concentrations in adults after prenatal exposure to famine. Br J Haematol 111, 112-117.

7. Juhola J, Magnussen CG, Viikari JS, et al. (2011) Tracking of serum lipid levels, blood pressure, and body mass index from childhood to adulthood: the Cardiovascular Risk in Young Finns Study. J Pediatr 159, 584-590.

8. Kaser S, Ebenbichler CF, Wolf HJ, et al. (2001) Lipoprotein profile and cholesteryl ester transfer protein in neonates. Metab Clin Exp 50, 723-728.

9. HAPO Study Cooperative Research Group (2009) Hyperglycemia Adverse Pregnancy Outcome (HAPO) Study associations with neonatal anthropometrics. Diabetes 58, 453-459.

10. Barjaktarovic M, Korevaar TIM, Gaillard R, et al. (2017) Childhood thyroid function, body composition and cardiovascular function. Eur J Endocrinol 177, 319-327.

11. Maslin K, Shaw V, Brown A, et al. (2021) What is known about the nutritional intake of women with Hyperemesis Gravidarum? A scoping review. Eur J Obstet Gynecol Reprod Biol 257, 76-83.

12. Veenendaal MV, van Abeelen AF, Painter RC, et al. (2011) Consequences of hyperemesis gravidarum for offspring: a systematic review and meta-analysis. BJOG 118, 1302-1313.

13. Koot MH, Grooten IJ, Sebert S, et al. (2017) Hyperemesis gravidarum, cardiometabolic risk factors in adolescents: a follow-up of the Northern Finland Birth Cohort 1986. BJOG: Int J Obstet Gynaecol 124, 1107-1114. 
14. Grooten IJ, Painter RC, Pontesilli M, et al. (2015) Weight loss in pregnancy and cardiometabolic profile in childhood: findings from a longitudinal birth cohort. BJOG 122, 1664-1673.

15. Ayyavoo A, Derraik JG, Hofman PL, et al. (2013) Severe hyperemesis gravidarum is associated with reduced insulin sensitivity in the offspring in childhood. J Clin Endocrinol Metab 98, 3263-3268.

16. Poeran-Bahadoer S, Jaddoe VWV, Gishti O, et al. (2020) Maternal vomiting during early pregnancy and cardiovascular risk factors at school age: the Generation R Study. J Dev Orig Health Dis 11, 118-126.

17. Royal College of Obstetricians and Gynaecologists (2016) The Management of Nausea and Vomiting of Pregnancy and Hyperemesis Gravidarum: RCOG Green-Top Guideline No. 69. https://www.rcog.org.uk/en/guidelinesresearch-services/guidelines/gtg69/ (accessed June 2016).

18. Grooten IJ, Koot MH, van der Post JA, et al. (2017) Early enteral tube feeding in optimizing treatment of hyperemesis gravidarum: the Maternal and Offspring outcomes after Treatment of HyperEmesis by Refeeding (MOTHER) randomized controlled trial. Am J Clin Nutr 106, 812-820.

19. Grooten IJ, Mol BW, van der Post JA, et al. (2016) Early nasogastric tube feeding in optimising treatment for hyperemesis gravidarum: the MOTHER randomised controlled trial (Maternal and Offspring outcomes after Treatment of HyperEmesis by Refeeding). BMC Childbirth 16, 22

20. Visser GH, Eilers PH, Elferink-Stinkens PM, et al. (2009) New Dutch reference curves for birthweight by gestational age. Early Hum Dev 85, 737-744.

21. Organization for Economic Cooperation and Development (OECD) (2021) Our Global Reach. https://www.oecd.org/ about/members-and-partners/ (accessed January 2022).

22. Koren G, Piwko C, Ahn E, et al. (2005) Validation studies of the Pregnancy Unique-Quantification of Emesis (PUQE) scores. J Obstet Gynaecol 25, 241-244.

23. National Institute for Public Health and the Environment (2020) NEVO Online Version 2019/6.0, RIVM, Bilthoven. https://nevoonline.rivm.nl/ (accessed October 2020).

24. National Institute for Public Health and the Environment (2020) Portie-Online Version 2020/1.4, RIVM, Bilthoven. https:// portie-online.rivm.nl/ (accessed October 2020).

25. National Institute for Public Health and the Environment Dutch National Food Consumption Survey 2007-2010 (2012). -https://www.rivm.nl/documenten/vcp-2007-2010-deel2-voedingsmiddelen-nevo-codes (accessed October 2020).

26. Brink L, Postma-Smeets A, Stafleu A, et al. (2020) Richtliinen Schijf van Vijf (Guidelines for The Wheel of Five). Den Haag: Stichting Voedingscentrum Nederland;. 182. https:// www.voedingscentrum.nl/Assets/Uploads/voedingscentrum/ Documents/Professionals/Schijf\%20van\%20Vijf/Richtlijnen\%20 Schijf\%20van\%20Vijf.pdf (accessed October 2020).

27. Geurts M, Toxopeus I, Van Rossum C, et al. (2016) MEMO. Achtergrondgegevens van referentievoedingen voor de Richtlijnen Schiifvan Viff 2016 (MEMO: background information on dietary intake references for The Wheel of Five Guidelines). Bilthoven: National Institute for Public Health and the Environment.

28. Noordam C, Rotteveel J \& Schroor EJ (2010) Werkboek Kinderendocrinologie (Workbook Pediatric Endocrinology). Amsterdam: VU Boekhandel.

29. Federation of Medical Specialists (2012) Schildklierfunctiestoornissen (Thyroid Disfunction). https://richtlijnen database.nl/richtlijn/schildklierfunctiestoornissen/schildklier functiestoornissen_-_korte_beschrijving.html (accessed October 2020).
30. Dean CR, Bierma H, Clarke R, et al. (2021) A patient-clinician James Lind Alliance partnership to identify research priorities for hyperemesis gravidarum. BMJ Open 11, e041254.

31. van Vliet R, Bink M, Polman J, et al. (2018) Patient preferences and experiences in hyperemesis gravidarum treatment: a qualitative study. J Pregnancy 2018, 5378502.

32. Ebrahimi N, Maltepe C, Bournissen F, et al. (2009) Nausea and vomiting of pregnancy: using the 24-hour Pregnancy-Unique Quantification of Emesis (PUQE-24) scale. J Obstet Gynaecol Can 31, 803-807.

33. Lacasse A, Rey E, Ferreira E, et al. (2008) Validity of a modified Pregnancy-Unique Quantification of Emesis and Nausea (PUQE) scoring index to assess severity of nausea and vomiting of pregnancy. Am J Obstet Gynecol 198, 71-e1.

34. Crozier SR, Inskip HM, Godfrey KM, et al. (2008) Dietary patterns in pregnant women: a comparison of food-frequency questionnaires and $4 \mathrm{~d}$ prospective diaries. Br J Nutr 99, 869-875.

35. Shim JS, Oh K \& Kim HC (2014) Dietary assessment methods in epidemiologic studies. Epidemiol Health 36, e2014009.

36. Bland JM \& Altman DG (1995) Multiple significance tests: the Bonferroni method. BMJ 310, 170.

37. Wilkins JT, Li RC, Sniderman A, et al. (2016) Discordance between apolipoprotein B and LDL-cholesterol in young adults predicts coronary artery calcification. J Am Coll Cardiol 67, 193-201.

38. Walldius G, Jungner I, Holme I, et al. (2001) High apolipoprotein B, low apolipoprotein A-I, and improvement in the prediction of fatal myocardial infarction (AMORIS study): a prospective study. Lancet 358, 2026-2033.

39. Agrawal A, Shrivastava J, Dwivedi R, et al. (2017) Assessment of serum apolipoprotein B and apolipoprotein A-1 and their ratio in healthy full term small for gestational age newborns. J Neonatal-Perinatal Med 10, 49-53.

40. Katragadda T, Mahabala RS, Shetty S, et al. (2017) Comparison of cord blood lipid profile in preterm small for gestational age and appropriate for gestational age newborns. J Clin Diagn Res 11, SC05-SC07.

41. Kharb S, Kaur R, Singh V, et al. (2010) Birth weight, cord blood lipoprotein and apolipoprotein levels in Indian newborns. Int J Prev Med 1, 29-33.

42. Bakker A, Weel J \& Wolthuis A (2015) Laboratoriumdiagnostiek bij kinderen: een praktische handleiding. (Laboratory Diagnostics in Children: a Practical Manual). Houten: Prelum Uitgevers.

43. Bays HE, Toth PP, Kris-Etherton PM, et al. (2013) Obesity, adiposity, and dyslipidemia: a consensus statement from the National Lipid Association. J Clin Lipidol 7, 304-383.

44. de Rooij SR, Painter RC, Roseboom TJ, et al. (2006) Glucose tolerance at age 58 and the decline of glucose tolerance in comparison with age 50 in people prenatally exposed to the Dutch famine. Diabetologia 49, 637-643.

45. Ravelli AC, van der Meulen JH, Michels RP, et al. (1998) Glucose tolerance in adults after prenatal exposure to famine. Lancet 351, 173-177.

46. Koh D, Hume R, Eisenhofer G, et al. (2015) Maternal and fetal factors which influence cord blood glucose levels in term infants delivered by cesarean section. J Périnat Med $\mathbf{4 3}$, 339-346.

47. Soeters MR \& Soeters PB (2012) The evolutionary benefit of insulin resistance. Clin Nutr 31, 1002-1007.

48. Dean C (2014) Helping women prepare for hyperemesis gravidarum. Br J Midwifery 22, 847-852.

49. Koren $G$ \& Maltepe C (2004) Pre-emptive therapy for severe nausea and vomiting of pregnancy and hyperemesis gravidarum. J Obstet Gynaecol 24, 530-533. 